\title{
Size and porosity effects on thermal conductivity of nanoporous material with an extension to nanoporous particles embedded in a host matrix
}

\author{
H. Machrafi*, G. Lebon
}

Université de Liège, Thermodynamics of Irreversible Processes, Allée du 6-Août, 17, 4000, Liège, Belgium

*Corresponding author: H.Machrafi@ulg.ac.be

\begin{abstract}
.
A formula for the effective thermal conductivity of nanoporous media is derived, following a thermodynamic approach. An extension to nanocomposites composed of a homogeneous matrix wherein porous nanoparticles are dispersed is proposed as well. The originality of the model is that it is based on extended irreversible thermodynamics, a theory specifically designed for sub-scaled systems. Two different situations are discussed, in the first one, nanoporous silicon with spherical porous inclusions of micro, meso and macro-dimension respectively is considered. The description is validated by comparison with experimental data and five other models. Analysis of the results shows an excellent agreement of our theoretical approach with experiments in the whole range of porous radii, from 2 to $100 \mathrm{~nm}$. In the second part of the work, thermal conductivity of porous silicon nanoparticles embedded in a germanium host matrix is investigated. The coupled influence of the pore and nanoparticles sizes is emphasized.
\end{abstract}

Keywords: nanoporous media, thermal conductivity, phonon scattering, extended thermodynamics

\section{Introduction}

The effect of porosity is to decrease considerably the thermal conductivity of crystalline materials, as is observed, for instance, in nanoporous silicon [1-5]. This lowering property has been widely exploited with the objective to reinforce thermal insulation in several systems as microsensors, integrated circuits, semiconductor devices. It has therefore fostered an increasing interest during the last decade. Thermal properties of nanoporous media are generally investigated by referring to, for instance, kinetic theory of phonons [3], molecular simulations [5], Monte Carlo simulations [6], two-component materials [7] or hydrodynamic models [8]. In this work, we take a different route and prefer a thermodynamic approach. To be explicit, we will base our study on extended irreversible thermodynamics (EIT) [9, 10], which is a formalism specifically dedicated to the treatment of micro and nano systems and highfrequency processes. For the sake of completeness, the present analysis will be compared to other theoretical models and experimental data.

Nanoporous materials are generally made out of a homogeneous matrix wherein nanopores are dispersed. In that respect, such systems are similar to nanocomposites, wherein nanoparticles are embedded in a matrix, the difference being that nanoparticles are replaced by nanopores. A great deal of papers have been devoted to the problem of heat transport in nanofluids and nanocomposites, see for instance the review paper by Michaeledis [11]. In particular, the problem of the overall heat conductivity of the system as a function of the volume fraction of the nanoparticles and their size has drawn much attention. Our purpose in the present work is twofold. Firstly, it is the purpose to study the role of porosity and the size of the pores on the heat conductivity of porous systems, like nanoporous silicon, via our thermodynamic formalism. Secondly, we examine, using the same approach, the influence of the presence of porous nanoparticles on the heat conductivity of nanocomposites; as illustration, nanoporous 
silicon particles dispersed in a germanium matrix are considered. The effect on the thermal conductivity of the size and volume fraction of nanoporous silicon as well as their porosity and pore sizes is investigated.

\section{Heat conductivity of nano-porous Si devices}

In a previous paper [12], we have studied the influence of the presence of nano inclusions on the effective thermal conductivity of nanocomposites. Our aim here is to extend, the type of approach made in [12] to nanoporous materials. As in reference [12], the system is simplified by homogenizing the heterogeneous original material with the resulting homogeneous system called the effective medium approach (EMA). This approach was initiated by Nan et al [13] and revisited by Minnich and Chen [14]. For simplicity, the nanopores are supposed to be filled with air.

The basic formula for the effective thermal conductivity coefficient of nanoporous $\mathrm{Si}$ is that derived by Maxwell $[15,16]$ to describe electrical conductivity of rigid particles in a fluid, which we have adapted here for porous materials, combining it with EIT as explained later on:

$$
k_{p S i}^{e f f}=k_{m} \frac{2 k_{m}+(1+2 \alpha) k_{p}+2 \varepsilon\left[(1-\alpha) k_{p}-k_{m}\right]}{2 k_{m}+(1+2 \alpha) k_{p}-\varepsilon\left[(1-\alpha) k_{p}-k_{m}\right]} .
$$

Symbol $\varepsilon$ stands for the porosity, $k_{p}$ for the thermal conductivity of the nanopores and $k_{m}$ for the bulk thermal conductivity of the host medium. Working in analogy with previous works on heat transport in nanocomposites [12,14], $\alpha$ represents a dimensionless parameter describing the interaction between the nanopores and the medium; it reads as [14]

$\alpha=R k_{m} / r_{p}$

where $r_{p}$ is the nanopore radius, $R$ the thermal boundary resistance coefficient given by [14]

$R=4 / C_{m} v_{m}+4 / C_{p} v_{p}$

with $C_{m}, v_{m}, C_{p}$ and $v_{p}$ representing the specific heat capacities and the phonons group velocities of the bulk medium and the nanopores, respectively. It is important to recall that relation (3) is valid for diffusive scattering of phonons at the interface pores-medium, which is the working assumption of the present work.

An expression for $k_{m}$ was established in the framework of the effective-medium approach [13,14], while $k_{p}$ will be derived in the framework of EIT in the case of nanopore inclusions in homogeneous matrices.

The thermal conductivity of the host matrix is given by the classical Boltzmann expression

$k_{m}=\left.\frac{1}{3}\left(C_{m} v_{m} \Lambda_{m}\right)\right|_{T_{r e f}}$.

$T_{\text {ref }}$ is the reference temperature, say the room temperature and $\Lambda_{m}$ the mean free path of phonons in the matrix. Within the matrix, the phonons experience phonon-phonon interactions and the mean free path $\Lambda_{m}$ is given by the Matthiessen rule:

$\frac{1}{\Lambda_{m}}=\frac{1}{\Lambda_{m, b}}+\frac{1}{\Lambda_{m, \text { coll }}}$ 
with $\Lambda_{m, b}$ designating the mean free path in the bulk and $\Lambda_{m, \text { coll }}$ the supplementary contribution due to the interactions at the nanopore-medium interface $[6,17]$ given by

$\Lambda_{m, \text { coll }}=4 r_{p}(1-\varepsilon) / 3 \varepsilon$

This expression differs by that proposed by Minning and Chen [14] by the presence of the factor $(1-\varepsilon)$ in the numerator. This is justified by the fact that in the limit of a high porosity $(\varepsilon=$ $O(1)$ ), the mean free path of phonons colliding with the pores is expected to tend to zero, while the bulk mean free path $\Lambda_{m, b}$, being a material property, remains of course constant.

Let us now focus on the derivation of the thermal conductivity $k_{p}$ of the nanopore. We propose the closed-form formula

$k_{p}=k_{p}^{0} f(K n)$

wherein $k_{p}^{0}$, expressed by the equivalent of relation (5), is the value of the thermal conductivity for air, namely

$k_{p}^{0}=\left.\frac{1}{3}\left(C_{p} v_{p} \Lambda_{p, b}\right)\right|_{T_{r e f}}$

The quantity $f(K n)$ in (7) is a correction factor that takes into account the nature, the shape and the size of the nanoparticles, $K n$ being the Knudsen number defined as the ratio of the mean free path $\Lambda_{p, b}$ of the phonons inside the nanopore and its radius $r_{p}$ :

$K n=\Lambda_{p, b} / r_{p}$

The correction factor $f(K n)$ will be determined within the framework of EIT. Let us briefly recall the main steps leading to the expression of $f(K n)$. We assume that heat transport is described by a set of variables identified as the heat flux $\left(\vec{q} \equiv Q^{(1)}\right.$, where the index (1) indicates the order of the flux), the flux of the heat flux $Q^{(2)}$ defined by $\partial Q^{(1)} / \partial t=-\vec{\nabla} \cdot Q^{(2)}$, and higher order fluxes, $\partial Q^{(n)} / \partial t=-\vec{\nabla} \cdot Q^{(n+1)}$. These fluxes are obeying a hierarchy of time evolution equations, which in their simplest approximation are linearized. Performing a Fourier transform leads finally to a wavelength-dependent $(\boldsymbol{k})$ thermal conductivity taking the form of a continued fraction expansion:

$$
k_{p}(\boldsymbol{k})=\frac{k_{p}^{0}}{1+\frac{\boldsymbol{k}^{2} l_{1}^{2}}{1+\frac{\boldsymbol{k}^{2} l_{2}^{2}}{1+\frac{\boldsymbol{k}^{2} l_{3}^{2}}{1+\cdots}}}}
$$

where $\boldsymbol{k}$ is the wavenumber vector and $l_{n}$ the mean free path associated with the heat flux of order $n$. We now select the latter as given path $l_{n}$ of order $n$ in terms of $n$ as $l_{n}^{2}=$ $l^{2}(n+1)^{2} /\left(4(n+1)^{2}-1\right)$, with $l$ identified as the mean free path independent of the order of approximation, this being a natural choice in phonon's kinetic theory [18]. By identifying $\boldsymbol{k}$ (which becomes a scalar in our one-dimensional case) as $k=2 \pi / r_{p}$ and using the definition (9) of the Knudsen number in expression (10) has the asymptotic limit [19] 
$k_{p}=\frac{3 k_{p}^{0}}{4 \pi^{2} K n^{2}}\left[\frac{2 \pi K n}{\arctan (2 \pi K n)}-1\right]$.

For more details, we refer the reader to section 4.7 in reference [9]. Note that the result (11) is only valid for a spherical geometry. In the case of cylindrical fibers for instance, a modified expression has to be used [20].

\section{Results and comparison with other models}

The above general considerations will be applied to spherical nano inclusions in a Si matrix, the void phase is supposed to be occupied by air. Assuming that the pores are filled with air is by no means limitative of the present approach. Indeed, replacing air by any other gas or liquid will not modify the structure of the model. The material phonon properties are listed in Table 1.

Table1: Phonon properties of bulk materials at room temperature $\left(T_{r e f}\right)$

\begin{tabular}{lcll}
\hline Material & $\begin{array}{l}\text { Heat Capacity } \\
{\left[\mathrm{MJ} / \mathrm{m}^{3} \mathrm{~K}\right]}\end{array}$ & $\begin{array}{l}\text { Group velocity } \\
{[\mathrm{m} / \mathrm{s}]}\end{array}$ & $\begin{array}{l}\text { Mean free path } \\
{[\mathrm{nm}]}\end{array}$ \\
\hline $\mathrm{Si}$ (dispersion) & $0.93^{\mathrm{a}}$ & $1804^{\mathrm{a}}$ & $268^{\mathrm{a}}$ \\
$\mathrm{Si}$ (Debye) & $1.66^{\mathrm{a}}$ & $6400^{\mathrm{a}}$ & $40.9^{\mathrm{a}}$ \\
& & & \\
$\mathrm{Ge}$ & $0.87^{\mathrm{a}}$ & $1042^{\mathrm{a}}$ & $171^{\mathrm{a}}$ \\
$\mathrm{Air}$ & $0.00121^{\mathrm{b}}$ & $347^{\mathrm{b}}$ & $185.8^{\mathrm{c}}$ \\
\hline
\end{tabular}

${ }^{\mathrm{a}}[21]$

b $[22]$

${ }^{\mathrm{c} C a l c u l a t e d}$ via $\Lambda_{p, b}=3 k /\left(C_{p} v_{p}\right)$, with $k=0.026 \mathrm{Wm}^{-1} \mathrm{~K}^{-1}[16]$

The values for $\mathrm{Si}$ and $\mathrm{Ge}$ are derived from the dispersion model, using Chen's terminology [21]. The values for Si listed on the second row correspond to the Debye model [21], used by one of the models to which our results will be compared. The final expression of the thermal conductivity of nanoporous Si sample is given by relation (1) with sub-indices $m$ and $p$ standing for bulk Si and spherical nanopores respectively.

The validity of our approach will be checked by comparing our results with experimental data and five different models [6, 8, 23, 24] briefly described below. The comparison with the aforementioned models will also allow better understanding the underlying difficulties in modeling thermal conductivity of nano-porous materials. The first model, referred to as Eucken's formula (e.g [6]), is a simplification of expression (1) wherein $k_{m}$ is replaced by the bulk value $k_{m}^{0}$, whereas $k_{p}$ is neglected:

$k_{p S i}^{\text {eff,Eucken }}=k_{m}^{0} \frac{2-2 \varepsilon}{2+\varepsilon}$.

The drawback of such a formulation is that it does not take into account the effects of particle size and phonons interactions.

A more sophisticated formalism, was developed by Alvarez et al [8] and also used by Criado-Sancho et al. [3,4]. It is based on a phonon hydrodynamic version of Stokes resistance force exerted on a sphere in an infinite medium. It was found that 
$k_{p S i}^{\text {eff }, \text { hydro }}=k_{m}^{0} \frac{1}{\frac{1}{(1-\varepsilon)^{3}}+\frac{9}{2} \varepsilon \frac{K n^{2}}{1+K n *\left(0.864+0.290 e^{-1.25 / K n}\right)}\left(1+\frac{3}{\sqrt{2}} \sqrt{\varepsilon}\right)}$

In this model, the particle size and mean free path of phonons are not considered in the bulk material, but only in the correcting factor through the Knudsen number.

The third model used for comparison consists in letting $K n$ in (13) tend to zero so that expression (14) reduces to the so-called percolation model,

$k_{p S i}^{\text {eff,perco }}=k_{m}^{0}(1-\varepsilon)^{3}$.

The fourth model, proposed by Lysenko et al [23], consists in modifying the percolation model formula by a correcting factor involving the mean free path of phonons in the bulk material and the pore radius via Knudsen's number $K n=\Lambda_{p, b} / \mathrm{r}_{p}$, namely

$k_{p S i}^{e f f, L y s}=\frac{k_{m}^{0}}{1+\frac{4}{3} K n}(1-\varepsilon)^{3}$.

In Eq. (15), the porosity dependence is given by the term $(1-\varepsilon)^{3}$, which means that the mean free path does not depend on the porosity as is the case in our model.

In another model, based on a formalism rather similar to the model by Lysenko et al., Sumirat et al. [22] write the effective thermal conductivity in the form

$k_{p S i}^{e f f, \text { Sum }}=k_{m}^{0} \frac{(1-\varepsilon)}{1+\varepsilon^{1 / 3} K n}$.

Comparison between our model and the five other ones expressed by relations (12), to (16) is found in Fig 1, wherein the effective thermal conductivity is plotted as a function of the porosity for a pore radius of $10 \mathrm{~nm}$. Whatever the selected model, the heat conductivity is decreasing with the porosity, this reduction is easily understood owing to the weak thermal conductivity of air enclosed in the pores. 


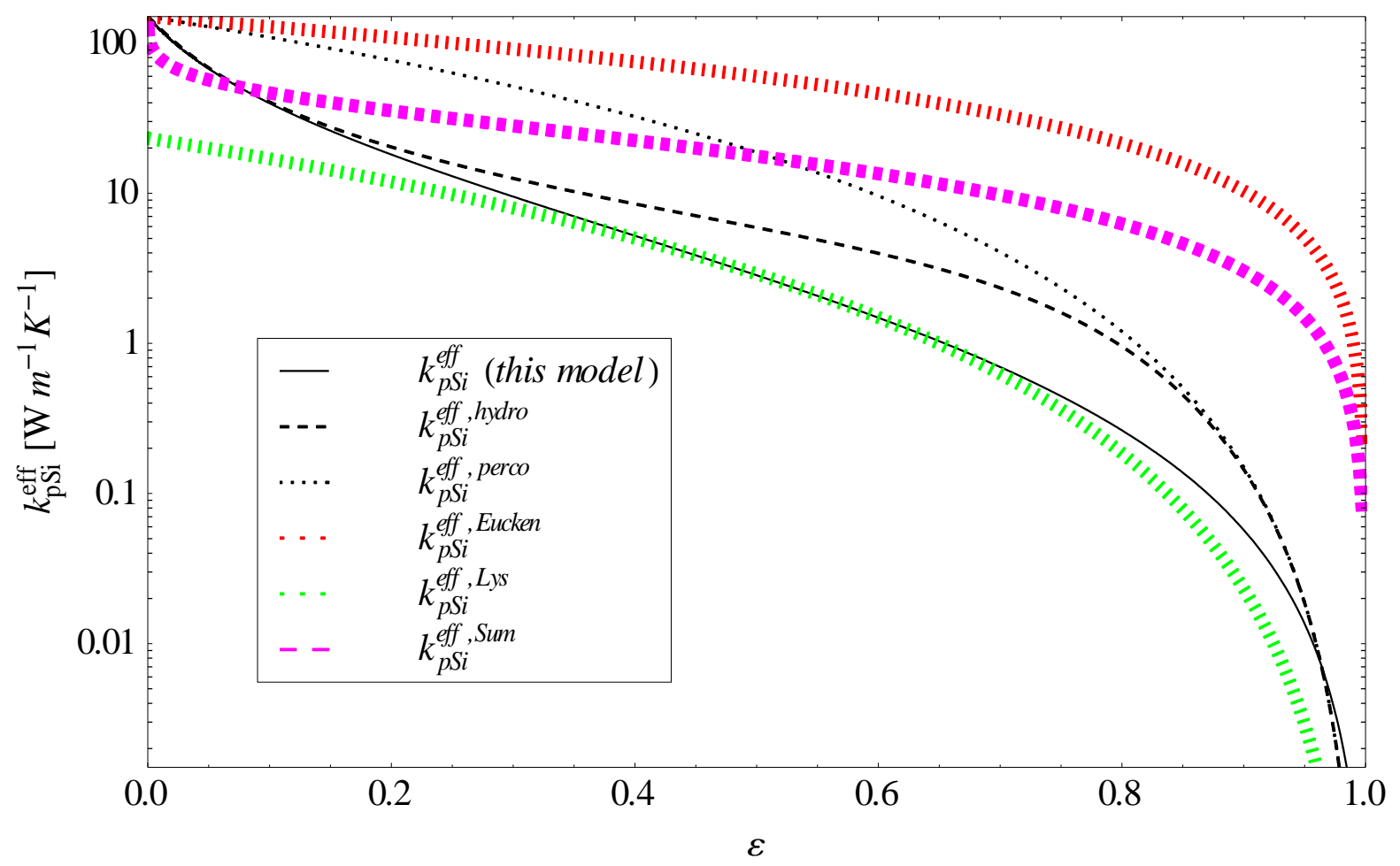

Fig. 1 Effective thermal conductivity as a function of porosity for a pore radius of $10 \mathrm{~nm}$. Comparison between our model and five other ones.

Our model is shown to be in satisfactory agreement with the hydrodynamic one, at values of porosity smaller than 0.2. In contrast, our results disagree with Eucken's approximation and the simplified formula (14), which predict overestimated results, particularly Eucken's approach. This is a clear indication that pore sizes play a decisive role in the calculations of thermal conductivity in nanoporous materials (an observation already well known for nanocomposites [12-14]). The hydrodynamic formulation does consider pore size and the ballistic property of the heat transport which is not the case of the simplified formula which express the heat conductivity exclusively as a function of the porosity. In the limit of very large porosity, the size is no longer relevant for any of the models and heat conductivity tends uniformly to that of air, at the exception of Eucken's. The results from Fig. 1 show that for small porosities, there is a rather good agreement between our model and the hydrodynamic one. This is explained by the fact that at small porosities, the collision mean free path in the bulk material (see Eq. (6)) is rather large so that the phonons travelling in the bulk are hardly influenced by the presence of the pores. However, at larger porosities, a clear discrepancy is visible. This can be interpreted by the particular treatment reserved in our model to the influence of the pore size and the mean free path which enter into the description of heat transport in both the bulk material and the nano-pores. At intermediate porosity range $(0.3<\varepsilon<0.7)$, a rather good agreement is found between our model and the "Lys" model [23]. This indicates that the dependence of the mean free path on nano-pore size and porosity (only considered in our model) becomes relevant at porosities larger than 0.7. At small porosities, however, the Lysenko model shows an important discrepancy with respect to ours and, more disturbing, when porosity tends to zero, the thermal conductivity does not approach the bulk value of silicon given by $k_{m}^{0}=150 \mathrm{Wm}^{-1} \mathrm{~K}^{-1}$. The formula (16) proposed by Sumirat et al [24] leads to results that are intermediate between Eucken's formula and the Lysenko model. The question that finally arises is, what is the best approach? 
Complementary information is provided by Table 2 wherein the various models are compared to experimental data.

Table 2: Thermal conductivities (in $\mathrm{Wm}^{-1} \mathrm{~K}^{-1}$ ) for different porosities and pore radii (in $\mathrm{nm}$ ) and comparison between the five models discussed in this section with experimental data

\begin{tabular}{ll|l|l|lllll}
\hline Porosity & Pore radius & Experiments & EIT & Hydro & Lys & Sum & Eucken & Perco \\
\hline $0.40^{\mathrm{a}}$ & $1-5$ & 1.2 & $0.6-2.7$ & $1.0-4.6$ & $0.6-2.7$ & $3-13$ & 75 & 32.4 \\
$0.40^{\mathrm{b}}$ & 100 & 31.2 & 32.0 & 29.1 & 20.9 & 69 & 75 & 32.4 \\
$0.50^{\mathrm{b}}$ & 10 & 3.9 & 2.8 & 5.9 & 2.9 & 17.6 & 60 & 18.7 \\
$0.60^{\mathrm{b}}$ & 10 & $2-5$ & 1.5 & 4.0 & 1.5 & 13.5 & 46 & 9.6 \\
$0.64^{\mathrm{c}}$ & 2 & 0.2 & 0.23 & 0.96 & 0.25 & 2.9 & 41 & 7.0 \\
$0.71^{\mathrm{c}}$ & 2 & 0.14 & 0.13 & 0.77 & 0.13 & 2.3 & 32 & 3.7 \\
$0.79^{\mathrm{c}}$ & 3 & 0.06 & 0.09 & 0.67 & 0.07 & 2.3 & 23 & 1.4 \\
$0.89^{\mathrm{c}}$ & 5 & 0.04 & 0.04 & 0.18 & 0.02 & 1.9 & 11 & 0.2 \\
\hline
\end{tabular}

${ }^{\mathrm{a}}[25]$

${ }^{\mathrm{b}}[1]$

c[7]

Table 2 indicates that whatever the model, the thermal conductivity decreases with the porosity and that at fixed porosity, it is considerably reduced by passing from macro to nanopores. It is worth to stress that our model fits rather well experiments for all the values of porosities considered. The hydrodynamic model predicts satisfactory the results at small porosities, but not at larger porosities. The Lysenko model is in agreement with experiments at intermediate porosities, except for a $100 \mathrm{~nm}$ pore radius. It is confirmed that the results provided by the Eucken, Sumirat and percolation models are rather far from the experimental data for all the ranges considered.

A further comparison between EIT, the hydrodynamic model and experimental data $[1,7,23]$ for several values of the pore radii $\left(r_{p}=2,5,10\right.$ and $\left.100 \mathrm{~nm}\right)$ is found in Fig. 2(a). The same comparison is made in Fig. 2(b), but instead of the hydrodynamic model, we compare with the Lysenko model.

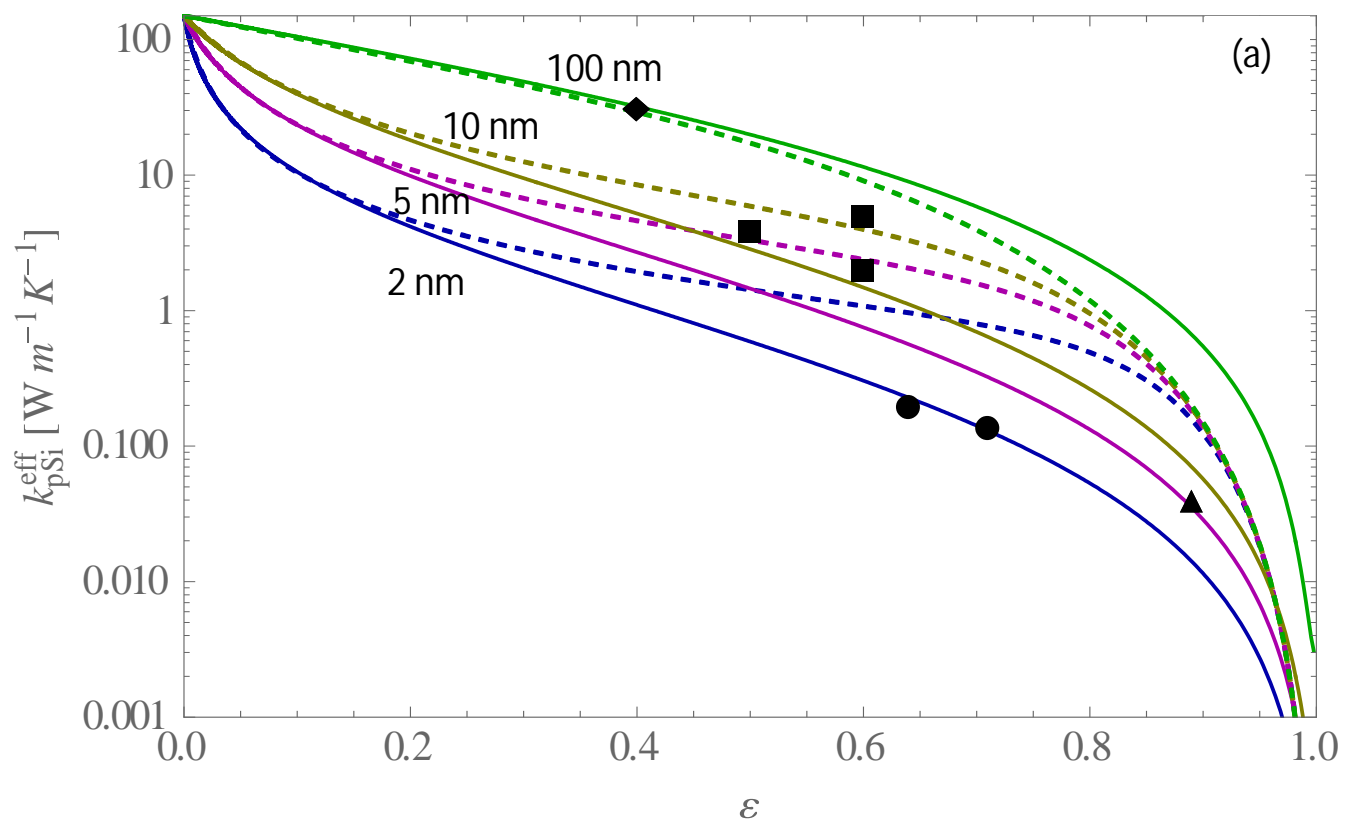




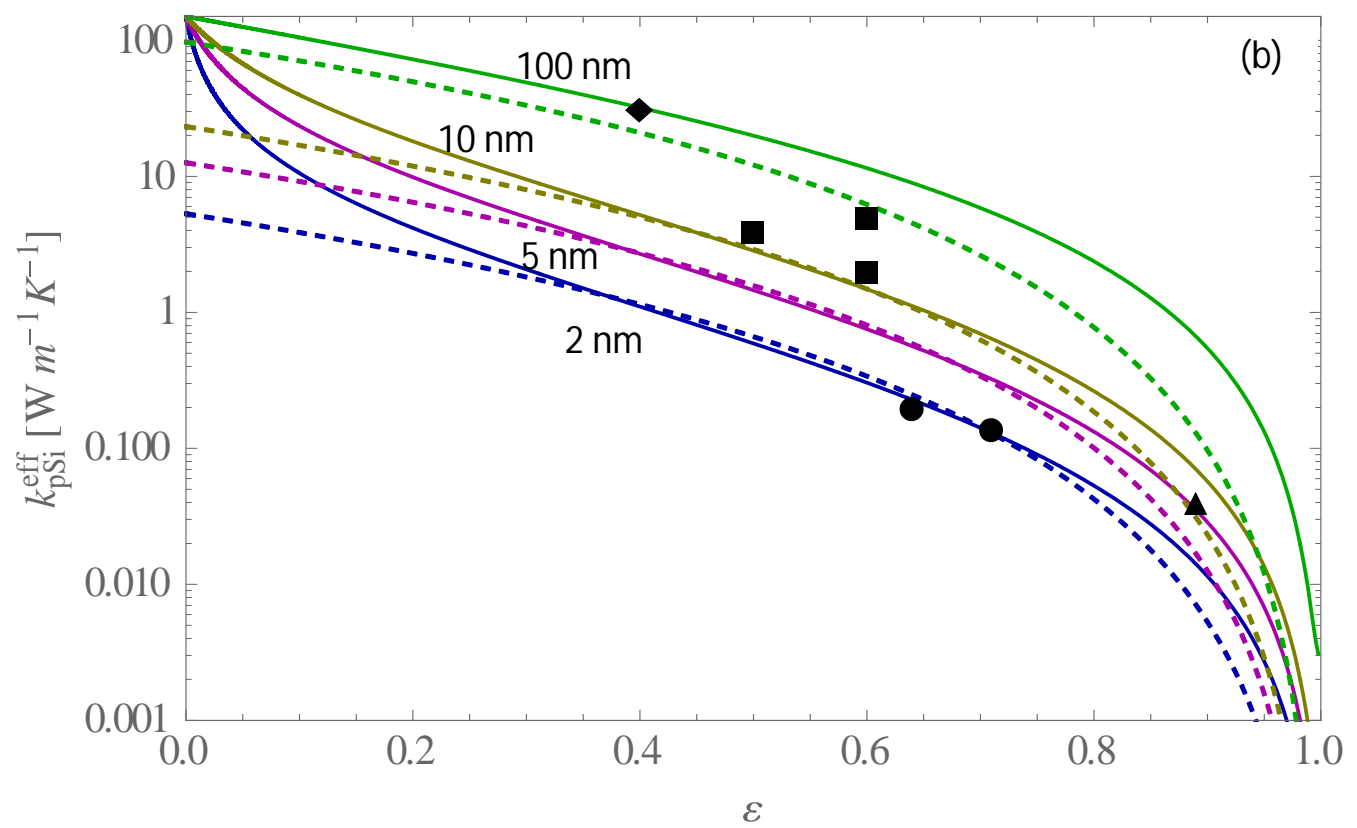

Fig. 2 Effective thermal conductivity $\left(\mathrm{Wm}^{-1} \mathrm{~K}^{-1}\right)$ as a function of porosity for pore radii of 2 $(\bullet), 5(\boldsymbol{\Delta}), 10(\boldsymbol{\bullet})$ and $100(\checkmark) \mathrm{nm}$. Comparison of the results of our model (solid lines) with reported experimental data [1,7] and, in dashed lines, the hydrodynamic model [8] (Fig. 2a) and the Lysenko model [23] (Fig. 2b).

A good agreement between our model and the experimental data is observed. There is only one point, at radius $10 \mathrm{~nm}$ and porosity 0.6 that seems to be in less satisfactory accord. However, it should be stressed that this point is experimentally not well defined. Indeed, the experimental values of the thermal conductivity at these values is found between 2 and $5 \mathrm{Wm}^{-}$ ${ }^{1} \mathrm{~K}^{-1}$, while our model predicts $1.5 \mathrm{Wm}^{-1} \mathrm{~K}^{-1}$. Recalling that the curve starts with a value of the thermal conductivity of $150 \mathrm{Wm}^{-1} \mathrm{~K}^{-1}$ (at zero porosity), the agreement may be considered as rather fair. Anyway, for all the other reported experimental points, the agreement with our model is better than with the five other discussed models. For porosities smaller than 0.6 and pore sizes of 10 and $100 \mathrm{~nm}$, (see Fig. 2(a)) a good agreement with experimental data is reached between our model (solid line) and the hydrodynamic one. Contrary to our model, the hydrodynamic model does not compare well experimentally at pore sizes of 2 and $5 \mathrm{~nm}$ at porosities larger than 0.6. The poor correspondence at larger pore sizes has been discussed earlier. The good correspondence observed at higher pore sizes is due to the fact that at larger pore sizes (macroporous media), the ballistic effects become less important and classical descriptions remain satisfactory. At intermediate porosities, the Lysenko model agrees well with our model and the experimental data, except at a pore radius of $100 \mathrm{~nm}$ (see Fig. 2(b)). It is concluded that only the EIT model seems to be in accord with the experiments in the whole range of porosities and pore sizes considered in this work.

We also observe that generally, at a fixed value of the porosity, the thermal conductivity decreases under a reduction of the radius of the pores. This is easily understandable as smaller pore radii correspond to an increase of the total pore-matrix interface, at a given value of porosity. This results in an increase of scattering of matrix phonons at the pore-matrix interface, and consequently a reduction of the effective thermal conductivity. However, Eq. (11) shows interestingly that this is not the only reason for the reduction of the effective thermal conductivity. Indeed, smaller pore radii also result into larger values of the Knudsen number, whence a smaller $k_{p}$-value according to equation (11). This means that the increase of phonon 
collisions inside the pores (not considered up to now), contributes further to the reduction of the effective thermal conductivity.

\section{A mathematical model for porous nanoparticles embedded within a matrix}

In a previous work [12], we have calculated the effective heat conductivity of $\mathrm{Si} / \mathrm{Ge}$ composites with silicon spherical nanoparticle embedded in a germanium matrix. Here, instead of bulk $\mathrm{Si}$ particles, we will consider nanoporous Si particles ( $\mathrm{p}-\mathrm{Si}$ ) by focusing on the effects of porosity. The idea is to evaluate how the combination of two types of nano-inclusions (nanopores in $\mathrm{Si}$ nanoparticles) affects the effective thermal conductivity. This calls for some modifications and adaptations to the model developed in section 2 .

Let us first determine the expression of the thermal conductivity of the p-Si nanoparticles of radius $a_{p}$ assumed to be dispersed (with a volume fraction $\varphi$ ) in a Ge matrix. In virtue of Eq. (11), it is given by

$k_{p-S i}=\frac{3 k_{p S i}^{0}}{4 \pi^{2} K n_{p}^{2}}\left[\frac{2 \pi K n_{p}}{\arctan \left(2 \pi K n_{p}\right)}-1\right]$,

The next step is to state precisely what represent the quantities $K n_{p}$ and $k_{p S i}^{0}$. The latter designates the thermal conductivity of nanoporous silicon ( $\mathrm{p}$-Si) particles (with porosity $\varepsilon$ and pore radius $r_{p}$ ) by taking into account the size effects of the nanopores but not those of the nanoparticles themselves, its expression is given by Eq. (1) $k^{e f f}$ or, to be explicit, $k_{p S i}^{0} \equiv k_{p S i}^{e f f}$. We turn now our attention to the expression of the Knudsen number $K n_{p}$ of the $\mathrm{p}-\mathrm{Si}$ nanoparticles, it is defined as $K n_{p}=\Lambda_{p-S i} / \mathrm{a}_{p}$, with $\Lambda_{p-S i}$ the mean free path of phonons in the $\mathrm{p}$-Si nanoparticles approximated by (using Matthiesen's rule),

$\frac{1}{\Lambda_{p-S i}}=\frac{1}{\Lambda_{p}}+\frac{1}{\Lambda_{\text {air }}}$

where the quantities $\Lambda_{\text {air }}$ and $\Lambda_{p}$ are the mean free paths in air and bulk silicon, respectively. It remains to determine the thermal conductivity of the Ge host matrix, $k_{m G e}$. It is given by relation (4) with $\Lambda_{m}$ still expressed by (5) but now, instead of (6), the collision mean free path will read as [14]

$\Lambda_{m G e, \text { coll }}=4 a_{p} / 3 \varphi$

The overall effective thermal conductivity is still given by relation (1) wherein the porosity $\varepsilon$ is replaced by the volume fraction $\varphi$ of $\mathrm{p}$-Si nanoparticles in $\mathrm{Ge}$, so that

$k_{p-S i, G e}^{e f f}=k_{m G e} \frac{2 k_{m G e}+\left(1+2 \alpha_{*}\right) k_{p-S i}+2 \varphi\left[\left(1-\alpha_{*}\right) k_{p-S i}-k_{m G e}\right]}{2 k_{m G e}+\left(1+2 \alpha_{*}\right) k_{p-S i}-\varphi\left[\left(1-\alpha_{*}\right) k_{p-S i}-k_{m G e}\right]}$.

The quantity $\alpha$ in (1) is now replaced by $\alpha_{*}$ defined by

$\alpha_{*}=R_{*} k_{m G e} / a_{p}$

the thermal boundary resistance coefficient $R_{*}$ being given by [14] 
$R_{*}=\frac{4}{C_{m G e} v_{m G e}}+\frac{4}{C_{p-S i} v_{p-S i}}$

wherein $C_{m G e}$ and $v_{m G e}$ are respectively the specific heat capacity and phonon group velocity corresponding to germanium. The specific heat capacity $C_{p-S i}$ and phonon group velocity $v_{p-S i}$ are supposed to be given by the following mean values

$C_{p-S i}=\varepsilon C_{a i r}+(1-\varepsilon) C_{p}$,

$v_{p-S i}=\varepsilon v_{\text {air }}+(1-\varepsilon) v_{p}$

where the sub index " $p$ " " refers to bulk silicon. Figs. 3 and 4 depict the dependence of the effective thermal conductivities of the nano composite $\mathrm{p}-\mathrm{Si} / \mathrm{Ge}$ versus the volume fraction $\varphi$ of the $\mathrm{p}$-Si particles for several porosities $\varepsilon$, pore radii $r_{p}$ and particle radii $a_{p}$. Note that $\varepsilon=0$ corresponds to bulk silicon nanoparticles embedded in germanium as studied in [12], whereas $\varepsilon=1$ describes nanoporous Ge samples [6].
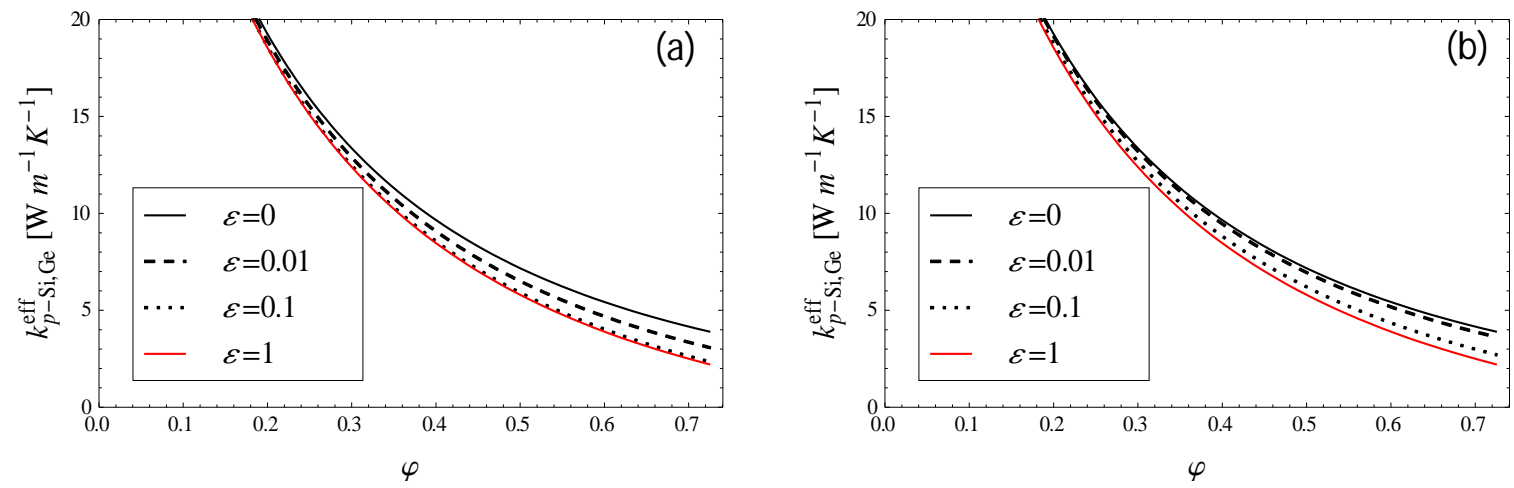

Fig. 3 Effective thermal conductivity as a function of the p-Si nanoparticle volume fraction, with (a) $r_{p}=1 \mathrm{~nm} \& a_{p}=25 \mathrm{~nm}$ and (b) $r_{p}=5 \mathrm{~nm} \& a_{p}=25 \mathrm{~nm}$, for several porosities.
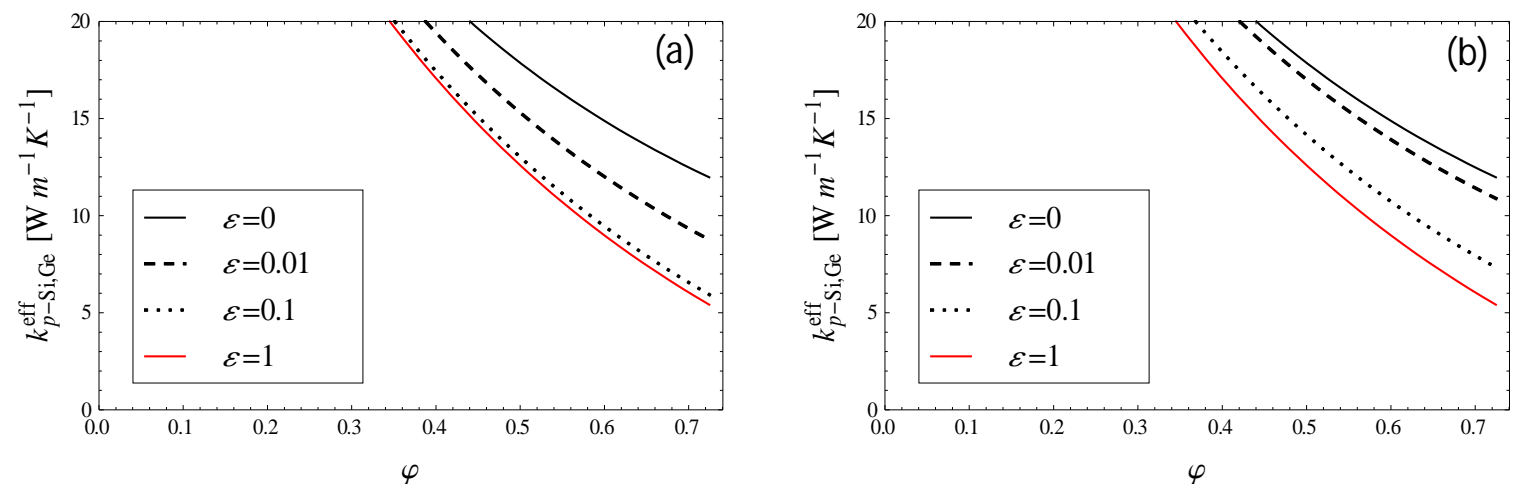

Fig. 4 Effective thermal conductivity as a function of the p-Si nanoparticle volume fraction, with (a) $r_{p}=1 \mathrm{~nm} \& a_{p}=100 \mathrm{~nm}$ and (b) $r_{p}=5 \mathrm{~nm} \& a_{p}=100 \mathrm{~nm}$, for several porosities.

Fig. 3(a) indicates that thermal conductivity is decreasing with increasing volume fraction of the porous p-Si nanoparticles. The effective heat conductivity is rather insensitive to the porosity of the $\mathrm{p}-\mathrm{Si}$ particles; indeed a porosity of 0.1 leads to results practically equivalent to fully porous particles with $\varepsilon=1$, which corresponds to the maximum isolation of the sample. By increasing the radius of the pores (from $r_{p}=1$ to $5 \mathrm{~nm}$ ) (see Fig. 3(b)), a value of $\varepsilon$ larger 
than 0.1 is necessary to reach the same result, though the thermal conductivity still remains rather insensitive to the porosity. This is however no longer true for large radii of the $\mathrm{p}-\mathrm{Si}$ nanoparticles $\left(a_{p}=100 \mathrm{~nm}\right)$ as shown by Fig. 4 . In this case, the reduction of the thermal conductivity is much larger when $\varepsilon$ is increased. The reduction is still reinforced by diminishing the size $r_{p}$ of the nanopores inside the p-Si particles. We can conclude that the behavior of the effective thermal conductivity is governed by the following four parameters: porosity, pore size, particle size and particle volume fraction. It appears that for small particle sizes $\left(a_{p} \leq 25 \mathrm{~nm}\right)$, porosity hardly influences the value of the thermal conductivity, whilst for large particle sizes $\left(a_{p} \geq 100 \mathrm{~nm}\right)$ porosity plays a more influential role.

\section{Conclusions}

The present paper comprises two parts. In the first one, the effect of the presence of nanopores on the thermal conductivity of silicon is investigated. This is achieved by referring to a thermodynamic formulation, namely extended irreversible thermodynamics, coupled to the socalled effective medium approximation. The role of porosity and size of the nanopores has been examined. The model has been validated against experimental data and compared to other theoretical models, being those of Stokes [8] and Eucken [6], two models presented recently by Lysenko et al [23] and Sumirat et al [24] and finally the simplified percolation model. It is shown that our model fits better the experimental values than the other models.

In the second part of this work, we extend the analysis to a nanocomposite of porous silicon nanoparticles embedded within a germanium matrix. This study generalizes the case wherein only bulk (without porosity) Si particles are considered. Emphasis is put on the influence on the effective thermal conductivity of $\mathrm{p}-\mathrm{Si} / \mathrm{Ge}$ composite of several parameters, as the particle size, pore size, porosity and particle volume fraction. The limiting cases are those of zero porosity (which corresponds to bulk Si nanoparticles in $\mathrm{Ge}$ ) and porosity tending to one (i.e. porous germanium).

The results that are obtained open interesting perspectives in the field of thermal properties of nanoporous semiconductors and are likely to be useful in the manufacturing of novel sensors or nanofuel cells [26, 27]. In relation with the subject discussed in this paper, a new domain of increasing interest is that of thermal rectifiers [28-30]. These are devices that are capable of transporting heat in one direction and blocking it in the opposite direction. They are analogous to diodes and transistors in electronics.

\section{Acknowledgements}

We thank BelSPo for financial support.

\section{References}

1. G. Benedetto, L. Boarino, and R. Spagnolo, Appl. Phys. A: Mater. Sci. Process., 64 (1997), p. 155.

2. U. Bernini, S. Lettieri, P. Maddalena, R. Vitiello, and G. D. Francia, J. Phys.C: Condens. Matter, 13 (2001), p. 1141.

3. M. Criado-Sancho, L.F. del Castillo, J. Casas-Vázquez, D. Jou, Phys. Lett. A, 376 (2012), p.1641.

4. M. Criado-Sancho, D. Jou, Phys. Lett. A, 377 (2013), p. 486.

5. J.-H. Lee, J. C. Grossman, J. Reed, and G. Galli, Appl. Phys. Lett. 91 (2007), p. 223110.

6. V. Jean, S. Fumeron, K. Termentzidis, S. Tutashkonko, and D. Lacroix, J. Appl. Phys. 115 (2014), p. 024304. 
7. G. Gesele, J. Linsmeier, V. Drach, J. Fricke, and R. Arens-Fischer, J. Phys. D. 30 (1997), p. 2911.

8. F. X. Alvarez, D. Jou, and A. Sellitto, Applied Physics Letters 97 (2010), p. 033103.

9. D. Jou, J. Casas-Vazquez and G. Lebon, Extended Irreversible Thermodynamics, (fourth edition) Springer, Berlin (2010).

10. G. Lebon, J. Non-Equilib. Thermodyn. 39 (2014), p. 35.

11. E.E. Michaeledis, J. Non-Equilib. Thermodyn. 38 (2013), p. 1.

12. H. Machrafi, G. Lebon, Int. J. Nanosc., doi: 10.1142/S0219581X14500227, (2014).

13. C.W. Nan, R. Birringer, D.R. Clarke and H. Gleiter, J. Appl. Phys. 81 (1997), p. 6692.

14. A. Minnich and G. Chen, Appl. Phys. Lett. 91 (2007), p. 073105.

15. J.C. Maxwell, Treatise on Electricity and Magnetism, (second edition) Clarendon, Oxford (1881).

16. D. Bruggeman, Anal. Phys. 24 (1935), p. 636.17. L.C. Liu and M.J.Huang, Int. J. Therm. Sc. 49 (2010), p. 1547.

18. W. Dreyer and H. Struchtrup, Continuum Mech. Thermodyn. 5 (1993), p. 3.

19. S. Hess, Z. Naturforsch. 32a (1977), p. 678.

20. G. Lebon, H. Machrafi, submitted for publication (2014).

21. G. Chen, Phys. Rev. B 57 (1998), p. 14958.

22. D.W. Green and R.H. Perry, Perry's Chemical Engineers' Handbook, (eighth edition) McGraw-Hill, New York, (2008).

23. V. Lysenko, Ph. Roussel, B. Remaki, G. Delhomme, A. Dittmar, D. Barbier, V. Strikha, C. Martelet, J. Porous Mater. 7 (2000), p. 177.

24. I. Sumirat, Y. Ando and S. Shimamura, J. Porous Mater. 13 (2006), p. 439.

25. A. Drost, P. Steiner, H. Moser, and W. Lang, Sens. Mater. 7 (1995), p. 111.

26. S. Ozdemir, J.L. Gole, Curr. Opin. Solid State Mater. Sci. 11 (2007), p. 92.

27. Y. Chen, Y. Lin, Y. Zhang, S. Wang, D. Su, Z. Yang, M. Han and F. Chen, Nano Energy 8 (2014), p. 25.

28. C. Dames, J. Heat Transf. 131 (2009), p. 061301.

29. N. Li, J. Ren, L. Wang, G. Zhang, P. Hanggi and B. Li, , Rev. Mod. Phys. 84 (2012), p. 1045.

30. M. Criado-Sancho, F.X Alvarez and D. Jou, J. Appl. Phys. 114 (2013), p. 053512. 


\section{Figure captions}

Fig. 1 Effective thermal conductivity as a function of porosity for a pore radius of $10 \mathrm{~nm}$. Comparison between our model and five other ones.

Fig. 2 Effective thermal conductivity $\left(\mathrm{Wm}^{-1} \mathrm{~K}^{-1}\right)$ as a function of porosity for pore radii of 2 $(\bullet), 5(\boldsymbol{\Delta}), 10(\boldsymbol{\bullet})$ and $100(\diamond) \mathrm{nm}$. Comparison of the results of our model (solid lines) with reported experimental data [1,7] and, in dashed lines, the hydrodynamic model [8] (Fig. 2a) and the Lysenko model [23] (Fig. 2b).

Fig. 3 Effective thermal conductivity as a function of the p-Si nanoparticle volume fraction, with (a) $r_{p}=1 \mathrm{~nm} \& a_{p}=25 \mathrm{~nm}$ and (b) $r_{p}=5 \mathrm{~nm} \& a_{p}=25 \mathrm{~nm}$, for several porosities.

Fig. 4 Effective thermal conductivity as a function of the p-Si nanoparticle volume fraction, with (a) $r_{p}=1 \mathrm{~nm} \& a_{p}=100 \mathrm{~nm}$ and (b) $r_{p}=5 \mathrm{~nm} \& a_{p}=100 \mathrm{~nm}$, for several porosities. 


\section{Tables}

Table1: Phonon properties of bulk materials at room temperature $\left(T_{\text {ref }}\right)$

\begin{tabular}{lcll}
\hline Material & $\begin{array}{c}\text { Heat Capacity } \\
{\left[\mathrm{MJ} / \mathrm{m}^{3} \mathrm{~K}\right]}\end{array}$ & $\begin{array}{l}\text { Group velocity } \\
{[\mathrm{m} / \mathrm{s}]}\end{array}$ & $\begin{array}{l}\text { Mean free path } \\
{[\mathrm{nm}]}\end{array}$ \\
\hline Si (dispersion) & $0.93^{\mathrm{a}}$ & $1804^{\mathrm{a}}$ & $268^{\mathrm{a}}$ \\
$\mathrm{Si}$ (Debye) & $1.66^{\mathrm{a}}$ & $6400^{\mathrm{a}}$ & $40.9^{\mathrm{a}}$ \\
& & & \\
$\mathrm{Ge}$ & $0.87^{\mathrm{a}}$ & $1042^{\mathrm{a}}$ & $171^{\mathrm{a}}$ \\
$\mathrm{Air}$ & $0.00121^{\mathrm{b}}$ & $347^{\mathrm{b}}$ & $185.8^{\mathrm{c}}$ \\
\hline
\end{tabular}

a[21]

$\mathrm{b}[22]$

${ }^{c}$ Calculated via $\Lambda_{p, b}=3 k /\left(C_{p} v_{p}\right)$, with $k=0.026 \mathrm{Wm}^{-1} \mathrm{~K}^{-1}[16]$

Table 2: Thermal conductivities (in $\mathrm{Wm}^{-1} \mathrm{~K}^{-1}$ ) for different porosities and pore radii (in $\mathrm{nm}$ ) and comparison between the five models discussed in this section with experimental data

\begin{tabular}{|c|c|c|c|c|c|c|c|c|}
\hline Porosity & Pore radius & Experiments & EIT & Hydro & Lys & Sum & Eucken & Perco \\
\hline $0.40^{\mathrm{a}}$ & $1-5$ & 1.2 & $0.6-2.7$ & $1.0-4.6$ & $0.6-2.7$ & $3-13$ & 75 & 32.4 \\
\hline $0.40^{\mathrm{b}}$ & 100 & 31.2 & 32.0 & 29.1 & 20.9 & 69 & 75 & 32.4 \\
\hline $0.50^{\mathrm{b}}$ & 10 & 3.9 & 2.8 & 5.9 & 2.9 & 17.6 & 60 & 18.7 \\
\hline $0.60^{\mathrm{b}}$ & 10 & $2-5$ & 1.5 & 4.0 & 1.5 & 13.5 & 46 & 9.6 \\
\hline $0.64^{\mathrm{c}}$ & 2 & 0.2 & 0.23 & 0.96 & 0.25 & 2.9 & 41 & 7.0 \\
\hline $0.71^{\mathrm{c}}$ & 2 & 0.14 & 0.13 & 0.77 & 0.13 & 2.3 & 32 & 3.7 \\
\hline $0.79^{\mathrm{c}}$ & 3 & 0.06 & 0.09 & 0.67 & 0.07 & 2.3 & 23 & 1.4 \\
\hline $0.89^{\mathrm{c}}$ & 5 & 0.04 & 0.04 & 0.18 & 0.02 & 1.9 & 11 & 0.2 \\
\hline
\end{tabular}

